\title{
Gudermannian neural networks using the optimization procedures of genetic algorithm and active set approach for the three-species food chain nonlinear model
}

\author{
Zulqurnain Sabir $^{1} \cdot$ Mohamed R. Ali $^{2,3} \cdot$ R. Sadat $^{4}$
}

Received: 7 May 2021 / Accepted: 1 December 2021

○ The Author(s), under exclusive licence to Springer-Verlag GmbH Germany, part of Springer Nature 2021

\begin{abstract}
The present study is to investigate the Gudermannian neural networks (GNNs) using the optimization procedures of genetic algorithm and active-set approach (GA-ASA) to solve the three-species food chain nonlinear model. The three-species food chain nonlinear model is dependent upon the prey populations, top-predator, and specialist predator. The design of an errorbased fitness function is presented using the sense of the three-species food chain nonlinear model and its initial conditions. The numerical results of the model have been obtained by exploiting the GNN-GA-ASA. The obtained results through the GNN-GA-ASA have been compared with the Runge-Kutta method to substantiate the correctness of the designed approach. The reliability, efficacy and authenticity of the proposed GNN-GA-ASA are examined through different statistical measures based on single and multiple neurons for solving the three-species food chain nonlinear model.
\end{abstract}

Keywords Gudermannian neural network · Three-dimensional food chain nonlinear model · Nonlinear differential system · Runge-Kutta scheme $\cdot$ Active-set algorithm $\cdot$ Statistical studies

\section{Introduction}

The study of two and three trophic-level based on food chain systems using the structure of logistic prey $X$, specialist Lotka-Volterra predator $Y$ and top-predator $Z$ (Freedman and Waltman 1977; Freedman and So 1985; Muratori and Rinaldi 1992; Kuznetsov and Rinaldi 1996; Rinaldi et al. 1996; El-Owaidy et al. 2001; Umar et al. 2019). The general

\section{R. Sadat}

r.mosa@zu.edu.eg

Zulqurnain Sabir

zulqurnain_maths@hu.edu.pk

Mohamed R. Ali

mohamed.reda@fue.edu.eg

1 Department of Mathematics and Statistics, Hazara University, Mansehra, Pakistan

2 Faculty of Engineering and Technology, Future University, Cairo, Egypt

3 Department of Basic Science, Faculty of Engineering at Benha, Benha University, Benha 13512, Egypt

4 Department of Physics and Engineering Mathematics, Faculty of Engineering, Zagazig University, Zagazig, Egypt form of the state system based on the three species food chain nonlinear model is written as (Aziz-Alaoui 2002):

$\left\{\begin{array}{l}X^{\prime}(T)=a_{0} X(T)-b_{0} X^{2}(T)-\frac{v_{0} X(T) Y(T)}{X(T)+d_{0}}, X(0)=l_{1}, \\ Y^{\prime}(T)=-a_{1} Y(T)+\frac{v_{1} X(T) Y(T)}{d_{1}+X(T)}-\frac{v_{2} Y(T) Z(T)}{Y(T)+d_{2}}, Y(0)=l_{2}, \\ Z^{\prime}(T)=c_{3} Z^{2}(T)-\frac{v_{3} Z^{2}(T)}{Y(T)+d_{3}}, Z(0)=l_{3} .\end{array}\right.$

The above system represents the three-dimensional food chain nonlinear model that has been investigated analytically/ numerically with the prey population $X$, which implemented as a single food predator $Y$ together with prey of a top-predator $Z$. The features of prey $X$ along with the species $Y$ present the modeling of Volterra scheme, which indicates the predator population reduces exponentially in the absence of prey. The association of species $Z$ together with its prey $Y$ is formed based on the Leslie-Gower scheme (Leslie and Gower 1960), which indicates the predator population reduces to the reciprocal of per capita availability of its most special food (Upadhyay et al. 1998). $l_{1}, l_{2}$ and $l_{3}$ are the positive initial conditions (Ics). The model parameter's detail of the three-dimensional food chain nonlinear model is expressed in the Table 1. 
Table 1 Illustrations of the three-dimensional food chain nonlinear model

\begin{tabular}{ll}
\hline Parameters & Specification \\
\hline$a_{0}$ & Prey growth rate $X$ \\
$b_{0}$ & Competition power among individuals-based species $X$ \\
$d_{2}$ & Elimination rate of Y per capita is $\frac{v_{2}}{2}$ \\
$d_{0}, d_{1}$ & Environment produce conservation to prey $X$ \\
$a_{1}$ & Rate at $Y$ will decrease in the omission of $X$ \\
$d_{3}$ & Surplus loss in the species of $Z$ due to severe insuffi- \\
& ciency of its selected food $Y$ \\
$c_{3}$ & Development rate of $Z$ \\
$v_{0}, v_{1}, v_{2}, v_{3}$ & $\begin{array}{l}\text { Obtained maximum values per capita by reducing the } X \\
l_{1}, l_{2}, l_{3}\end{array}$ \\
\hline
\end{tabular}

The stochastic computing processes have been executed to solve a large variety of nonlinear systems, few of them are fractional singular systems (Sabir et al. 2021c, d, e), like higher order singular systems (Ayub et al. 2021; Sabir et al. 2021a), dengue fever system (Umar et al. 2020b, c, d, e), SITR based COVID-19 models (Umar et al. 2020b, c, d, e, 2021a, b), delay singular function system (Khan et al. 2021; Sabir et al. 2021b), SIR system for spreading infection and treatment (Umar et al. 2021a, b), mosquito release system in the heterogeneous environment (Umar et al. 2020a), doubly singular nonlinear models (Raja et al. 2019; Sabir et al. 2020a, b, c), rank-constrained spectral clustering ( $\mathrm{Li}$ et al. 2018a, b), zero-shot event detection system (Li et al. 2019), fuzzy K-means clustering associated discriminative embedding scheme (Li et al. 2018a, b), multiclass classification systems (Yan et al. 2020). dynamic affinity graph construction strategy for spectral clustering (Nie et al. 2020), enhanced multilayer piezoelectric transducer design (Naz et al. 2021), performance investigation of the heat sink of functionally graded material of the porous fin (Ahmad et al. 2021), impact of heat transfer in a Bodewadt flow model in a set of Eq. (1). A brief summary of innovative insights and contributions of the presented study is listed in terms of salient features as follows:

- A novel application of artificial intelligent knacks via Gudermannian neural networks (GNNs) models optimized with genetic algorithm and active-set approach (GA-ASA), i.e., GNNs-GA-ASA is introduced to solve a mathematical model of the three-species food chain nonlinear systems (TS-FCNS).

- The design of an error-based fitness function is effectively portrayed for TS-FCNS for the dynamics of the prey populations, top-predator and specialist predator.

- The numerical results of the TS-FCNS have been obtained by exploiting computation heuristics of GNNsGA-ASA and comparison with the outcomes of the Runge-Kutta method substantiated the correctness of the designed approach.

- The reliability, efficacy and authenticity of the proposed GNNs-GA-ASA are further scrutinized through different statistical measures based on single and multiple executions for solving the three-species food chain nonlinear model.

The paper is organized as: Sect. 2 defines the computational procedures based on GNNs-GA-ASA along with the statistical measures are provided in the next section. The result and discussion are provided in Sect. 3. The concluding remarks and future research directions are provided in Sect. 4.

\section{Designed procedures: GNNs-GA-ASA}

The mathematical formulations of the three-dimensional food chain nonlinear model together with derivatives are derived as:

$$
\begin{gathered}
{[\hat{X}(T), \hat{Y}(T), \hat{Z}(T)]=\left[\begin{array}{l}
\sum_{q=1}^{s} r_{X, q} M\left(w_{X, q} T+n_{X, q}\right), \sum_{q=1}^{s} r_{Y, q} M\left(w_{Y, q} T+n_{Y, q}\right), \\
\sum_{q=1}^{s} r_{Z, q} M\left(w_{Z, q} T+n_{Z, q}\right),
\end{array}\right],} \\
{\left[\hat{X}^{\prime}(T), \hat{Y}^{\prime}(T), \hat{Z}^{\prime}(T)\right]=\left[\begin{array}{l}
\sum_{q=1}^{s} r_{X, q} M^{\prime}\left(w_{X, q} T+n_{X, q}\right), \sum_{q=1}^{s} r_{Y, q} M^{\prime}\left(w_{Y, q} T+n_{Y, q}\right), \\
\sum_{q=1}^{s} r_{Z, q} M^{\prime}\left(w_{Z, q} T+n_{Z, q}\right),
\end{array}\right],}
\end{gathered}
$$

(Awais et al. 2021), thin film flow model over a stretched surface (Uddin et al. 2021) and state estimation problems arising in underwater Markov chain maneuvering targets (Ali et al. 2021). All these utmost applications inspired the authors to explore/exploit/investigate artificial intelligencebased computational solver to solve the governing model of three-species food chain nonlinear model as presented where, $\mathrm{W}$ indicates an unidentified weight vector, given as: $W=\left[W_{X}, W_{Y}, W_{Z}\right]$, for $W_{X}=\left[r_{X}, \omega_{X}, n_{X}\right], W_{Y}=\left[r_{Y}, \omega_{Y}, n_{Y}\right]$, and $W_{Z}=\left[r_{Z}, \omega_{Z}, n_{Z}\right]$, where 
$r_{X}=\left[r_{X, 1}, r_{X T, 2}, r_{X, 3}, \ldots, r_{X, s}\right], r_{Y}=\left[r_{Y, 1}, r_{Y, 2}, r_{Y, 3}, \ldots, r_{Y, s}\right] r_{Z}=\left[r_{Z, 1}, r_{Z, 2}, r_{Z, 3}, \ldots, r_{Z, s}\right]$

$w_{X}=\left[w_{X, 1}, w_{X T, 2}, w_{X, 3}, \ldots, w_{X, s}\right], w_{Y}=\left[w_{Y, 1}, w_{Y, 2}, w_{Y, 3}, \ldots, w_{Y, s}\right] w_{Z}=\left[w_{Z, 1}, w_{Z, 2}, w_{Z, 3}, \ldots, w_{Z, s}\right]$

$n_{X}=\left[n_{X, 1}, n_{X, 2}, n_{X, 3}, \ldots, n_{X, s}\right], n_{Y}=\left[n_{Y, 1}, n_{Y, 2}, n_{Y, 3}, \ldots, n_{Y, s}\right] n_{Z}=\left[n_{Z, 1}, n_{Z, 2}, n_{Z, 3}, \ldots, n_{Z, s}\right]$

A merit function, i.e., Gudermannian function $M(\Psi)=2 \tan ^{-1}[\exp (\Psi)]-\frac{1}{2} \pi($ Sabir et al. $2021 \mathrm{c}, \mathrm{d}, \mathrm{e})$ is used as
Genetic algorithm is known as a famous, optimization global search scheme implemented to solve the linear/nonlinear models. It is performed to tackle both constrained/ unconstrained systems using the typical selection processes.

$$
\begin{aligned}
&= {\left[\begin{array}{l}
\sum_{k=1}^{r} q_{X, k}\left(2 \tan ^{-1} e^{\left(w_{X, k} \Psi+n_{X, k}\right)}-\frac{\pi}{2}\right), \sum_{k=1}^{r} q_{Y, k}\left(2 \tan ^{-1} e^{\left(w_{Y, k} \Psi+n_{Y, k}\right)}-\frac{\pi}{2}\right), \\
\sum_{k=1}^{r} q_{Z, k}\left(2 \tan ^{-1} e^{\left(w_{Z, k} \Psi+n_{Z, k}\right)}-\frac{\pi}{2}\right)
\end{array}\right], } \\
& {\left[\hat{X}^{\prime}(\Psi), \hat{Y}^{\prime}(\Psi), \hat{Z}^{\prime}(\Psi)\right]=\left[\begin{array}{l}
\sum_{k=1}^{r} 2 q_{X, k} w_{X, k}\left(\frac{e^{\left(w_{X, k} \Psi+n_{X, k}\right)}}{1+\left(e^{\left(w_{X, k} \Psi+n_{X, k}\right)}\right)^{2}}\right), \\
\sum_{k=1}^{r} 2 q_{Y, k} w_{Y, k}\left(\frac{e^{\left(w_{Y, k} \Psi+n_{Y, k}\right)}}{1+\left(e^{\left(w_{Y, k} \Psi+n_{Y, k}\right)}\right)^{2}}\right), \\
\sum_{k=1}^{r} 2 q_{Z, k} w_{Z, k}\left(\frac{e^{\left(w_{Z, k} \Psi+n_{Z, k}\right)}}{1+\left(e^{\left(w_{Z, k} \Psi^{\Psi}+n_{Z, k}\right)}\right)^{2}}\right)
\end{array}\right], }
\end{aligned}
$$

The merit function is provided as:

$\xi_{f}=\xi_{f-1}+\xi_{f-2}+\xi_{f-3}+\xi_{f-4}$

$\xi_{f-1}=\frac{1}{N} \sum_{j=1}^{N}\left[\hat{X}_{j}^{\prime}+a_{0} \hat{X}_{j}+b X_{j}^{2}+\frac{v_{0} \hat{X}_{j} \hat{Y}_{j}}{d_{0}+\hat{X}_{j}}\right]^{2}$,

$\xi_{f-2}=\frac{1}{N} \sum_{j=1}^{N}\left[\hat{Y}_{j}^{\prime}+a \widehat{Y}_{j}-\frac{v_{1} \hat{Y}_{j} \hat{Y}_{j}}{d_{1}+\hat{X}_{j}}+\frac{v_{2} \hat{Y}_{j} \hat{Z}_{j}}{d_{2}+\hat{Y}_{j}}\right]^{2}$,

$\xi_{f-3}=\frac{1}{N} \sum_{j=1}^{N}\left[\widehat{Z}_{j}^{\prime}-C_{3} \widehat{Z}_{j}+\frac{v_{3} \widehat{Z}_{j}}{d_{3}+\widehat{Y}_{j}}\right]^{2}$,

$\xi_{f-4}=\frac{1}{3}\left[\left(\widehat{X}_{0}-l_{1}\right)^{2}+\left(\hat{Y}_{0}-l_{2}\right)^{2}+\left(\widehat{Z}_{0}-l_{3}\right)^{2}\right]$,

where $\hat{X}_{j_{i}}=X\left(T_{j}\right), \hat{Y}_{j}=Y\left(T_{j}\right), \hat{Z}_{j}=Z(T J), N h=1$, and $T_{j}=h J . \widehat{X}_{j}, \widehat{Y}_{j}$ and $\widehat{Z}_{j}$ indicate the proposed results of the system (1). Likewise, the Eqs. (5)-(7) represent an error function of the three-dimensional food chain nonlinear model and its ICs.

\subsection{Optimization: GNN-GA-ASA}

This section indicates the optimization procedures to solve the three-dimensional food chain nonlinear model using the stochastic procedures based on GNN-GA-ASA.
GA is usually applied to regulate the results of the accurate population to solve the numerous complex/steep models of ideal training. Recently, GA is implemented in the brain tumor images (Simi et al. 2020), hospitalization expenditure systems (Tao et al. 2019), Thomas-Fermi model (Sabir et al. 2018), feature diversity in cancer microarray (Sayed et al. 2019), radiation protective in the bismuth-borate glasses (Wilson 2019), nonlinear electric circuit models (Mehmood et al. 2020), heat conduction model (Raja et al. 2018), HIV infection model (Umar et al. 2020b, c, d, e), wire coating with Oldroyd 8-constant fluid model (Munir et al. 2019), prediction differential system (Sabir et al. 2020a, b, c), periodic differential model (Sabir et al. 2020a, b, c) and cloud service optimization procedures (Yang et al. 2019). ASA is applied in pricing American better-of option on two assets (Gao et al. 2020), pressure-dependent models of water distribution systems with flow controls (Piller et al. 2020), nonlinear optimization with polyhedral constraints (Hager and Tarzanagh 2020), numerical solution of the optimal control problem governed by partial differential equation (Azizi et al. 2020), electrodynamic frictional contact problems (Abide et al. 2021) and quadratic semidefinite program with general constraints (Shen et al. 2021). The optimization process-based GA-ASA is applied to control the slowness of GA.

\subsection{Performance indices}

The performance through statistics based on the semi-interquartile range (S.I.R), mean absolute deviation (MAD), variance account for (VAF) and Theil's inequality coefficient 
(TIC) along with the global representation are observed to solve the three-dimensional food chain nonlinear model, given as:

$\left\{\right.$ S.I.R $=-0.5\left(Q_{1}-Q_{3}\right)$,

$\left\{Q_{1} Q_{3}\right.$ are the 1 st 3 rd quartiles,

$\left[\mathrm{MAD}_{X}, \mathrm{MAD}_{Y}, \mathrm{MAD}_{Z}\right]$

$$
=\left[\sum_{J=1}^{n}\left|X_{j}-\hat{X}_{j}\right|, \sum_{J=1}^{n}\left|Y_{j}-\hat{Y}_{j}\right|, \sum_{J=1}^{n}\left|Z_{j}-\widehat{Z}_{j}\right|\right]
$$

\section{Results and discussions}

The simplified form of the three-dimensional food chain nonlinear model using suitable parameter values is given as:

$\left\{\begin{array}{l}X^{\prime}(T)=1.5 X(T)-0.06 X^{2}(T)-\frac{X(T) Y(T)}{10+X(\chi)}, X_{0}=1.2, \\ Y^{\prime}(T)=-Y(T)+\frac{2 X(T) Y(T)}{10+X(T)}-\frac{0.405 Y(T) Z(T)}{10+Y(T)}, Y_{0}=1.2, \\ Z^{\prime}(T)=1.5 Z^{2}(T)-\frac{Z^{2}(T)}{20+Y(T)}, Z_{0}=1.2 .\end{array}\right.$

An objective function using the three-dimensional food chain nonlinear model is written as:

$\xi_{f}=\frac{1}{N} \sum_{J=1}^{N}\left(\begin{array}{l}{\left[\hat{X}_{J}^{\prime}-1.5 \hat{X}_{J}+0.06 \hat{X}_{J}^{2}+\frac{\hat{X}_{J} \hat{Y}_{J}}{10+\hat{X}_{J}}\right]^{2}+\left[\hat{Y}_{J}^{\prime}+\widehat{Y}_{J}-\frac{2 \hat{X}_{J} \hat{Y}_{J}}{10+\hat{X}_{J}}+\frac{0.405 \hat{Y}_{J} \hat{Z}_{J}}{10+Y_{J}}\right]^{2}} \\ +\left[\hat{Z}_{J}^{\prime}-1.5 \hat{Z}_{J}^{2}+\frac{\hat{Z}_{J}^{2}}{20+\hat{Y}_{J}}\right]^{2}\end{array}\right)$

$+\frac{1}{3}\left[\left(\widehat{X}_{0}-1.2\right)^{2}+\left(\hat{Y}_{0}-1.2\right)^{2}+\left(\widehat{Z}_{0}-1.2\right)^{2}\right]$

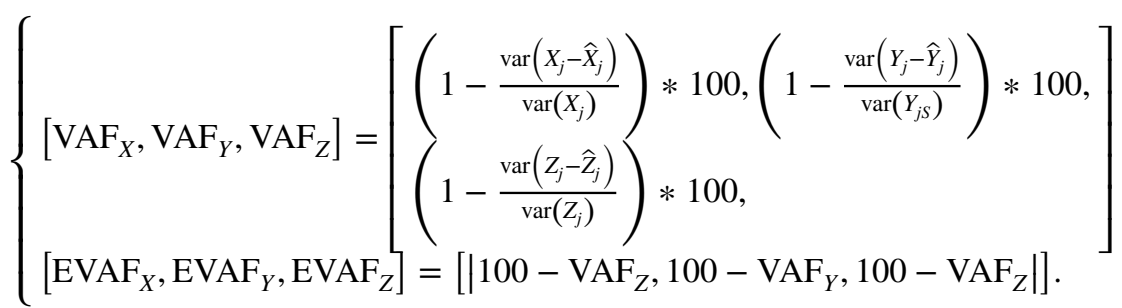

$\left[\mathrm{TIC}_{X}, \mathrm{TIC}_{Y}, \mathrm{TIC}_{Z}\right]$

$=\left[\begin{array}{l}\frac{\sqrt{\frac{1}{n} \sum_{J=1}^{n}\left(X_{j}-\hat{X}_{j}\right)^{2}}}{\left(\sqrt{\frac{1}{n} \sum_{J=1}^{n} X_{j}^{2}}+\sqrt{\frac{1}{n} \sum_{J=1}^{n} \hat{X}_{j}^{2}}\right)}, \frac{\sqrt{\frac{1}{n} \sum_{J=1}^{n}\left(Y_{j}-\hat{Y}_{j}\right)^{2}}}{\left(\sqrt{\frac{1}{n} \sum_{J=1}^{n} Y_{j}^{2}}+\sqrt{\frac{1}{n} \sum_{J=1}^{n} \hat{Y}_{j}^{2}}\right)}, \\ \frac{\sqrt{\frac{1}{n} \sum_{J=1}^{n}\left(Z_{j}-\hat{Z}_{j}\right)^{2}}}{\left(\sqrt{\frac{1}{n} \sum_{J=1}^{n} Z_{j}^{2}}+\sqrt{\frac{1}{n} \sum_{J=1}^{n} \hat{Z}_{j}^{2}}\right)},\end{array}\right]$,

where $\hat{X}, \widehat{Y}$ and $\widehat{Z}$ are the proposed solutions.
The mathematical results of the stochastic procedures based on GNN-GA-ASA:

$$
\begin{aligned}
\widehat{X}(\Psi) & =-4.1215\left(2 \tan ^{-1} e^{(-1.2073 \Psi+2.0489)}-0.5 \pi\right)-1.1601\left(2 \tan ^{-1} e^{-0.3909 \Psi-0.3808)}-0.5 \pi\right) \\
& -0.0100\left(2 \tan ^{-1} e^{(1.7292 \Psi+0.6374)}-0.5 \pi\right)-0.6757\left(2 \tan ^{-1} e^{(1.0278 \Psi-0.1355)}-0.5 \pi\right) \\
& -3.7274\left(2 \tan ^{-1} e^{(-1.3829 \Psi+3.4601)}-0.5 \pi\right)-0.6447\left(2 \tan ^{-1} e^{(1.3228 \Psi+1.3535)}-0.5 \pi\right) \\
& -1.6067\left(2 \tan ^{-1} e^{(-0.3206 \Psi-0.8162)}-0.5 \pi\right)+0.3827\left(2 \tan ^{-1} e^{(0.4292 \Psi+1.0041)}-0.5 \pi\right) \\
& 3.8605\left(2 \tan ^{-1} e^{(0.1557 \Psi+2.6613)}-0.5 \pi\right)+3.8418\left(2 \tan ^{-1} e^{(0.1831 \Psi+2.4362)}-0.5 \pi\right),
\end{aligned}
$$




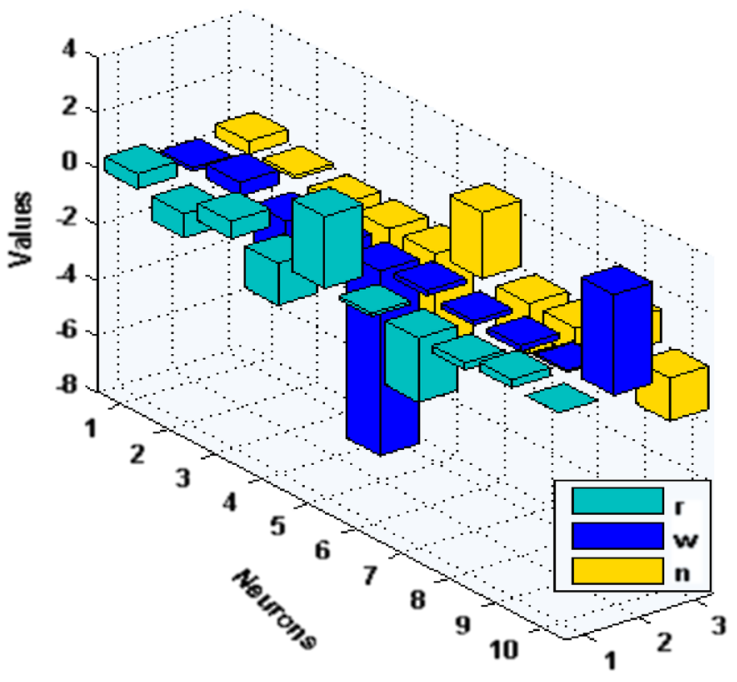

a Best weights: $X(T)$

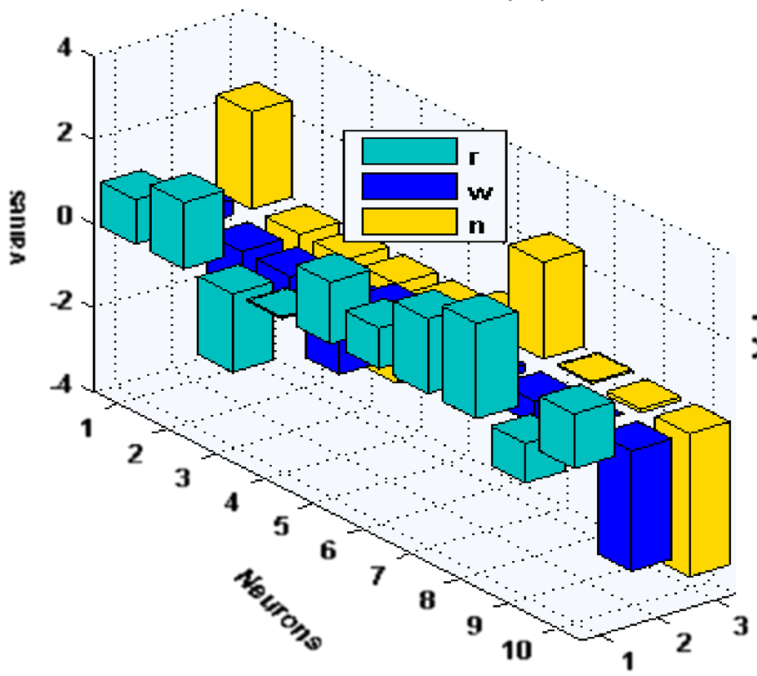

b Best weights: $Y(T)$

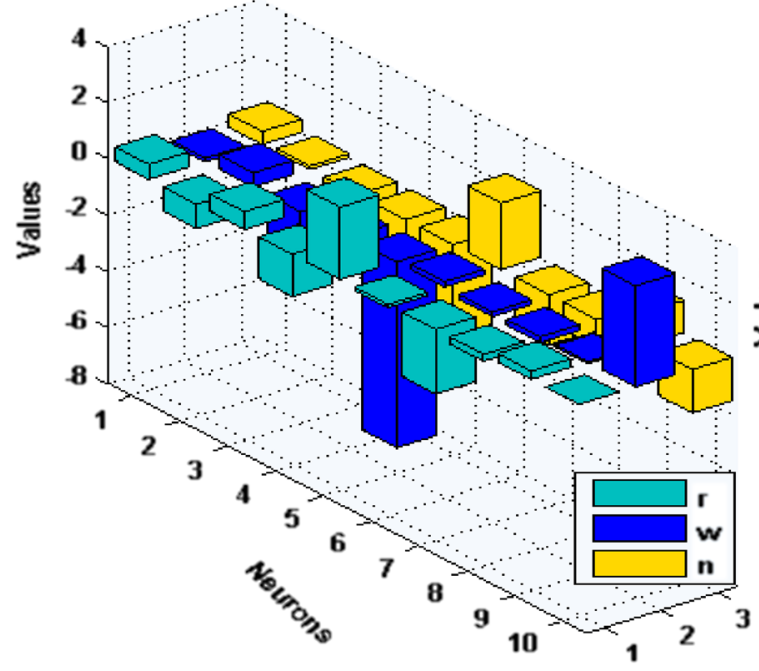

c Best weights: $Z(T)$
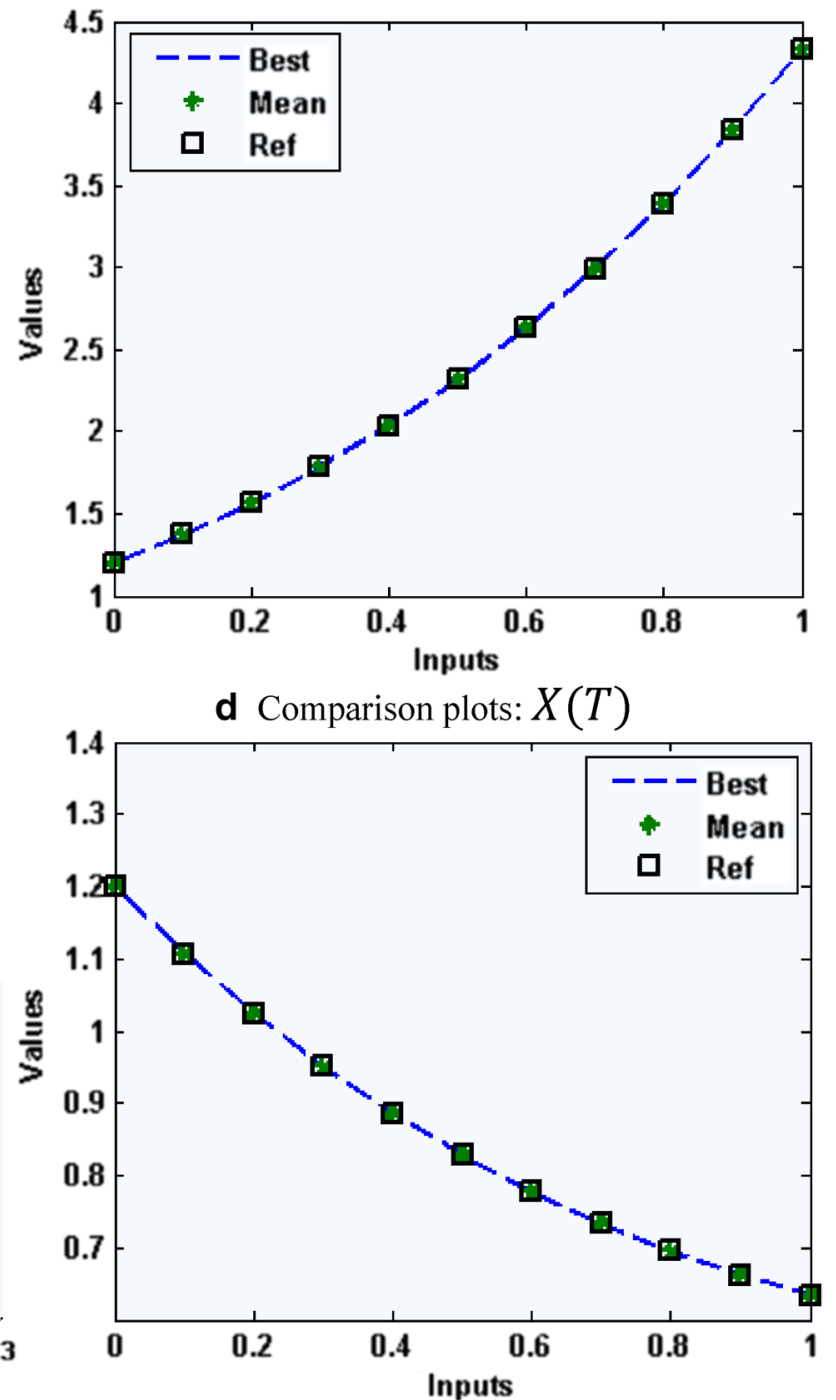

e Comparison plots: $Y(T)$

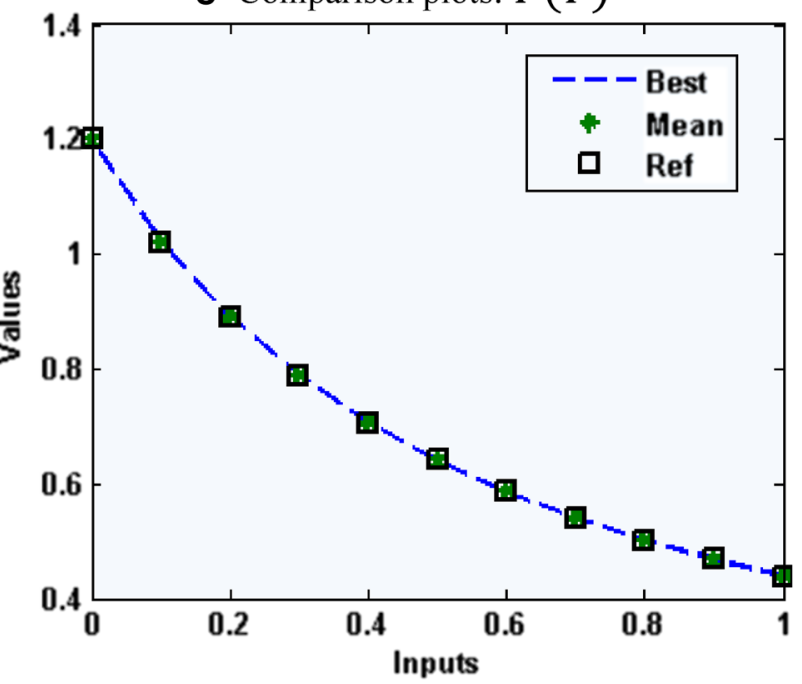

f Comparison plots: $Z(T)$

Fig. 1 Comparison of the results and best weight vectors for the three-dimensional food chain nonlinear model 
Fig. 2 AE values and the performances based on MAD, TIC and EVAF for the three-dimensional food chain nonlinear model

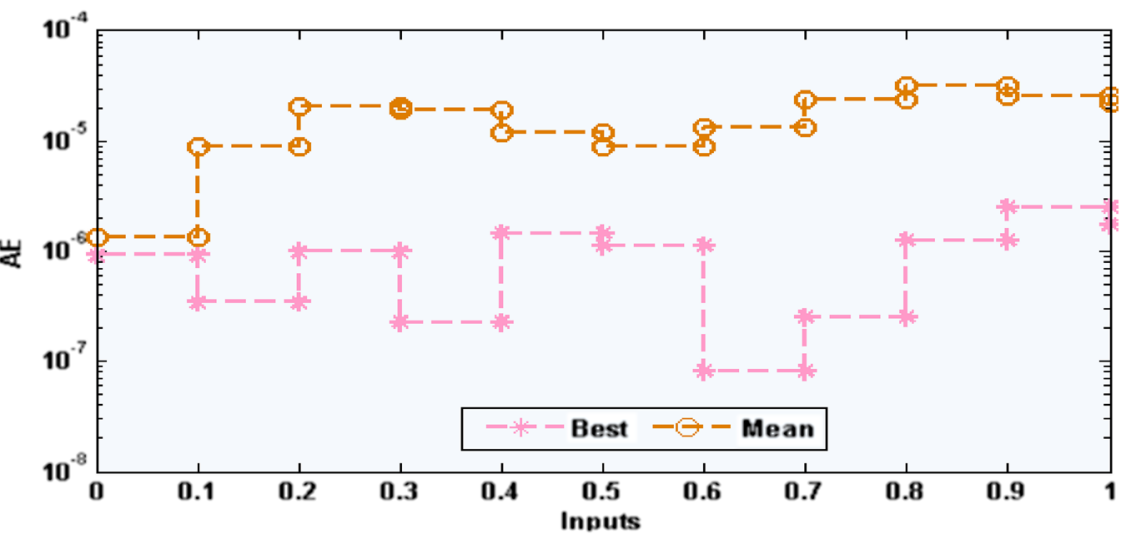

(a) AE: $X(T)$ values

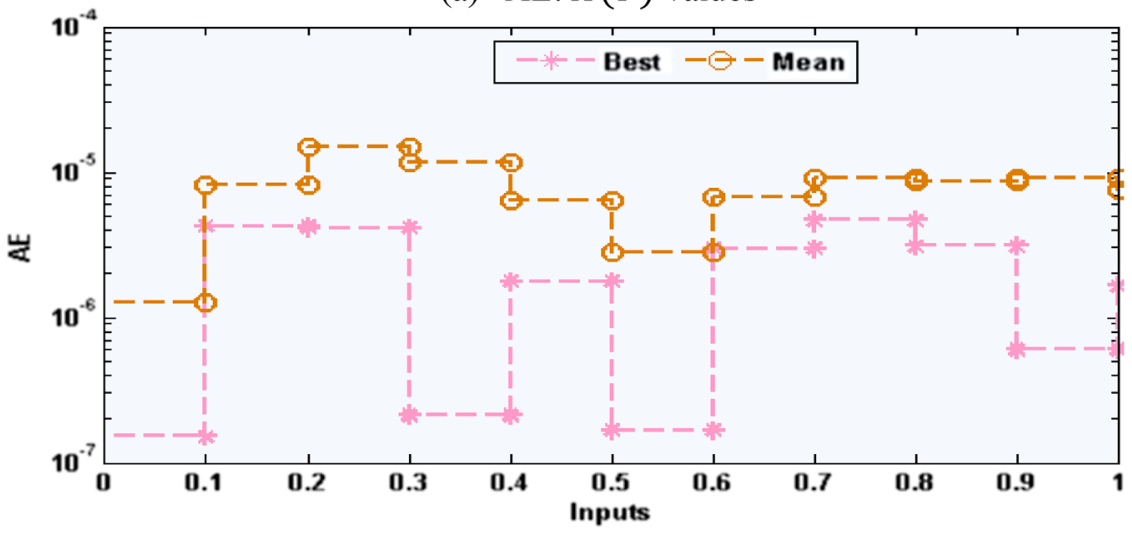

(b) AE: $Y(T)$ values

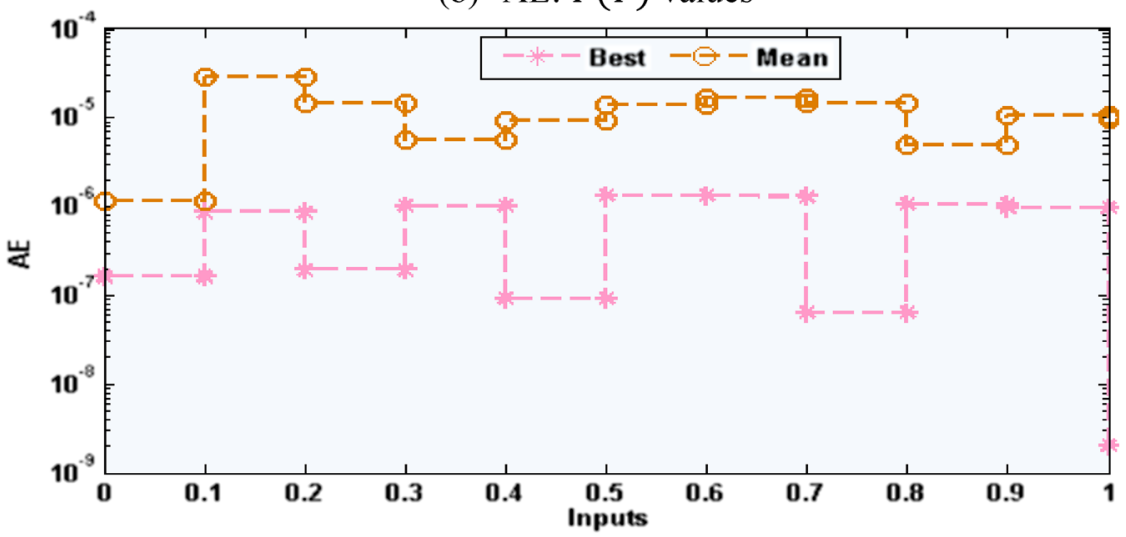

(c) AE: $Z(T)$ values

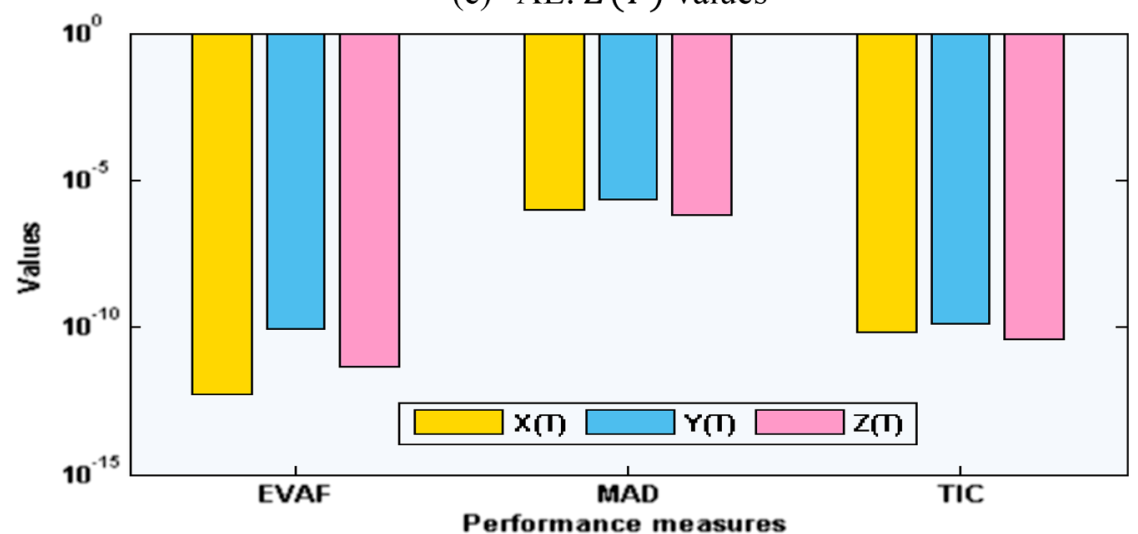


Fig. 3 TIC performances along with the boxplots based GNNGA-ASA for three-dimensional food chain nonlinear model

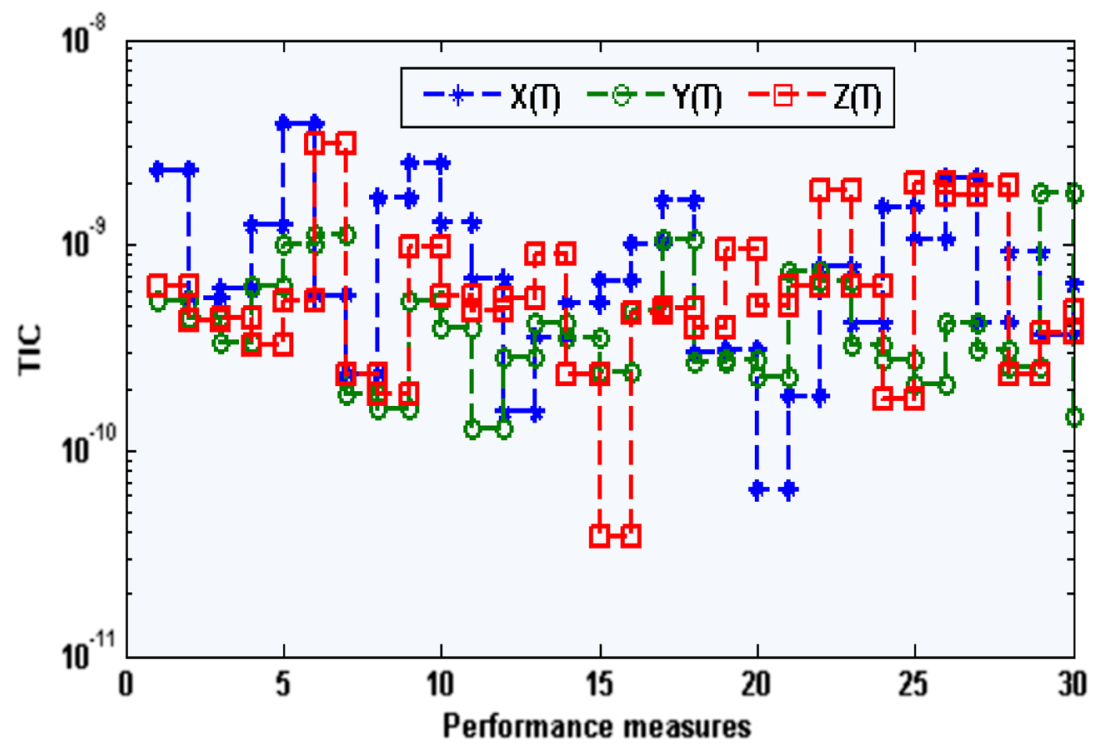

TIC performance operator for three-dimensional food chain nonlinear model

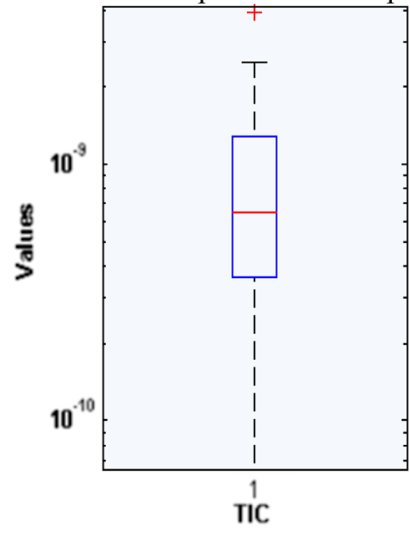

a Boxplots: $X(T)$ values

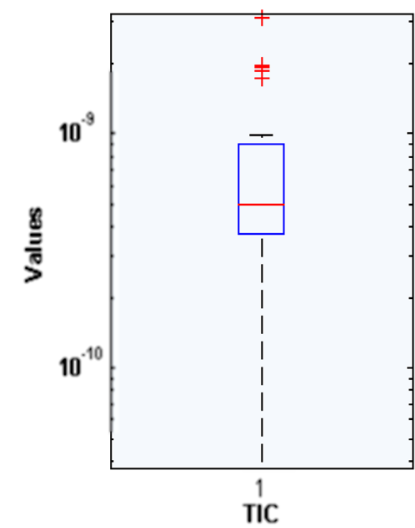

b Boxplots: $Y(T)$ values

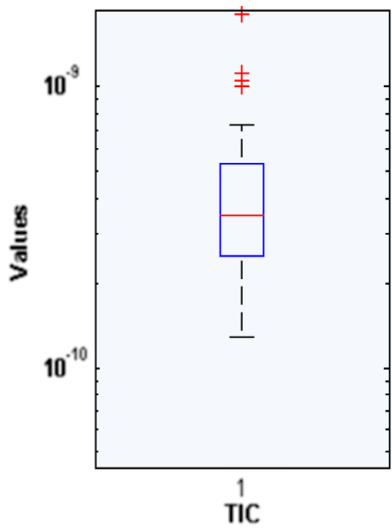

C Boxplots: $Z(T)$ values

$$
\begin{aligned}
\hat{X}(\Psi) & =1.0561\left(2 \tan ^{-1} e^{(0.3584 \Psi+2.3141)}-0.5 \pi\right)+1.5786\left(2 \tan ^{-1} e^{(-1.0463 \Psi-0.6359)}-0.5 \pi\right) \\
& -1.8630\left(2 \tan ^{-1} e^{(-0.6936 \Psi-0.3845)}-0.5 \pi\right)-0.0488\left(2 \tan ^{-1} e^{(-1.7367 \Psi-2.3627)}-0.5 \pi\right) \\
& 1.4624\left(2 \tan ^{-1} e^{(0.3281 \Psi-0.6821)}-0.5 \pi\right)+0.9619\left(2 \tan ^{-1} e^{(0.2794 \Psi+0.3296)}-0.5 \pi\right) \\
& 1.7775\left(2 \tan ^{-1} e^{(-0.1763 \Psi+2.3006)}-0.5 \pi\right)+2.2604\left(2 \tan ^{-1} e^{(-0.5332 \Psi+0.0478)}-0.5 \pi\right) \\
& -0.9345\left(2 \tan ^{-1} e^{(0.0191 \Psi-0.0942)}-0.5 \pi\right)+1.3007\left(2 \tan ^{-1} e^{(-2.8534 \Psi-3.4033)}-0.5 \pi\right),
\end{aligned}
$$

$$
\begin{aligned}
\widehat{X}(\Psi) & =0.5342\left(2 \tan ^{-1} e^{(0.1335 \Psi+0.4637)}-0.5 \pi\right)-0.8565\left(2 \tan ^{-1} e^{(0.5032 \Psi+0.1273)}-0.5 \pi\right) \\
& 0.6277\left(2 \tan ^{-1} e^{(-2.2469 \Psi-0.7075)}-0.5 \pi\right)-1.4959\left(2 \tan ^{-1} e^{(-1.1810 \Psi-3.0498)}-0.5 \pi\right) \\
& 2.5696\left(2 \tan ^{-1} e^{(-6.6073 \Psi-3.6405)}-0.5 \pi\right)-0.1184\left(2 \tan ^{-1} e^{(0.1933 \Psi+2.3627)}-0.5 \pi\right) \\
& -2.2969\left(2 \tan ^{-1} e^{(-0.1593 \Psi-1.7907)}-0.5 \pi\right)-0.2461\left(2 \tan ^{-1} e^{(-0.2011 \Psi-0.9760)}-0.5 \pi\right) \\
& 0.2974\left(2 \tan ^{-1} e^{(-0.0698 \Psi-1.1331)}-0.5 \pi\right)+0.0016\left(2 \tan ^{-1} e^{(3.5979 \Psi-1.5463)}-0.5 \pi\right),
\end{aligned}
$$


Figures 1, 2 and 3 illustrates the best weight vectors, result comparisons and the values of $\mathrm{AE}$ to solve the threedimensional food chain nonlinear model using the stochastic procedures based on GNNs-GA-ASA. The best weight values are illustrated in the three-dimensional food chain nonlinear model in Fig. 1a-c for 30 variables or 10 neurons. These weight vectors are established in Eqs. (15-17). The comparative performance of the results for the threedimensional food chain nonlinear model is illustrated in Fig. 1d-f. The plots of the AE have been established in Fig. $2 a-c$ for the three-dimensional food chain nonlinear model. The statistical operator plots along with the performances of the boxplots are illustrated in Fig. 3 to solve the three-dimensional food chain nonlinear model. The convergence measures are plotted using the TIC, MAD and EVAF to solve the three-dimensional food chain nonlinear model. The complexity of GNNs-GA-ASA in terms of execution time consumed for learning of the weights of the networks is calculated and it is found in the close vicinity of $30 \pm 10$ for the single runs of the algorithm.

\section{Conclusions}

This study aims to investigate the Gudermannian neural networks (GNNs) using the optimization procedures of genetic algorithm and active-set approach (GA-ASA) to solve the three-species food chain nonlinear model. An error function is constructed using the three classes of the threespecies food chain nonlinear model names as prey populations, top-predator and specialist predator and its initial conditions. The exactness of the scheme GNN-GA-ASA is observed by comparing the proposed results and the reference Runge-Kutta results to solve the three-dimensional food chain nonlinear model. The AE values are found in good measures to solve the three-dimensional food chain nonlinear model, i.e. around $10^{-05}-10^{-07}$. The performances of the operators TIC, EVAF and MAD proved the good illustrations to solve the three-dimensional food chain nonlinear model. The statistical Mean, S.I.R, Min, Max, MED and STD performances for 30 independent runs validate the correctness of the proposed stochastic procedures based on GNN-GA-ASA. Furthermore, the global performances through statistical trials of MED and S.I.R have been competently applied to solve the three-dimensional food chain nonlinear model.

In the future, the proposed stochastic procedures based on GNN-GA-ASA are accomplished to solve the environmental economic systems (Kiani et al. 2021; Nisar et al. 2021), information security models (Masood et al.2019, 2020, 2021) and fluid dynamic models (Awan et al. 2020, 2021; Raja et al. 2020; Umar et al. 2020b, c, d, e).
Funding Not applicable.

Data availability No data is used to support this study.

\section{Declarations}

Conflict of interest There is no conflict of interest. All authors contributed equally.

\section{References}

Abide S, Barboteu M, Cherkaoui S, Danan D, Dumont S (2021) Inexact primal-dual active set method for solving elastodynamic frictional contact problems. Comput Math Appl 82:36-59

Ahmad I, Ilyas H, Raja MAZ, Khan Z, Shoaib M (2021) Stochastic numerical computing with Levenberg-Marquardt backpropagation for performance analysis of heat sink of functionally graded material of the porous fin. Surf Interfaces 26:101403

Ali W, Li Y, Raja MAZ, Khan WU, He Y (2021) State estimation of an underwater markov chain maneuvering target using intelligent computing. Entropy 23(9):1124

Awais M, Bibi M, Raja MAZ, Awan SE, Malik MY (2021) Intelligent numerical computing paradigm for heat transfer effects in a Bodewadt flow. Surf Interfaces 26:101321

Awan SE, Raja MAZ, Mehmood A, Niazi SA, Siddiqa S (2020) Numerical treatments to analyze the nonlinear radiative heat transfer in MHD nanofluid flow with solar energy. Arab J Sci Eng 45(6):4975-4994

Awan SE, Raja MAZ, Gul F, Khan ZA, Mehmood A, Shoaib M (2021) Numerical computing paradigm for investigation of micropolar nanofluid flow between parallel plates system with impact of electrical MHD and Hall current. Arab J Sci Eng 46(1):645-662

Ayub A, Sabir Z, Altamirano GC et al (2021) Characteristics of melting heat transport of blood with time-dependent cross-nanofluid model using Keller-Box and BVP4C method. Eng Comput. https://doi.org/10.1007/s00366-021-01406-7

Aziz-Alaoui MA (2002) Study of a Leslie-Gower-type tritrophic population model. Chaos Solitons Fractals 14(8):1275-1293

Azizi M, Amirfakhrian M, Araghi MAF (2020) A fuzzy system based active set algorithm for the numerical solution of the optimal control problem governed by partial differential equation. Eur J Control 54:99-110

El-Owaidy HM, Ragab AA, Ismail M (2001) Mathematical analysis of a food-web model. Appl Math Comput 121(2):155-167

Freedman HI, So JWH (1985) Global stability and persistence of simple food chains. Math Biosci 76(1):69-86

Freedman HI, Waltman P (1977) Mathematical analysis of some threespecies food-chain models. Math Biosci 33(3):257-276

Gao Y, Song H, Wang X, Zhang K (2020) Primal-dual active set method for pricing American better-of option on two assets. Commun Nonlinear Sci Numer Simul 80:104976

Hager WW, Tarzanagh DA (2020) A Newton-type active set method for nonlinear optimization with polyhedral constraints. arXiv preprint arXiv:2011.01201

Khan I, Raja MAZ, Khan MAR et al (2021) Design of backpropagated intelligent networks for nonlinear second-order Lane-Emden pantograph delay differential systems. Arab J Sci Eng. https://doi.org/ 10.1007/s13369-021-05814-1

Kiani AK, Khan WU, Raja MAZ, He Y, Sabir Z, Shoaib M (2021) Intelligent backpropagation networks with bayesian regularization for mathematical models of environmental economic systems. Sustainability 13(17):9537 
Kuznetsov YA, Rinaldi S (1996) Remarks on food chain dynamics. Math Biosci 134(1):1-33

Leslie PH, Gower JC (1960) The properties of a stochastic model for the predator-prey type of interaction between two species. Biometrika 47(3-4):219-234

Li Z, Nie F, Chang X, Nie L, Zhang H, Yang Y (2018a) Rank-constrained spectral clustering with flexible embedding. IEEE Trans Neural Netw Learn Syst 29(12):6073-6082

Li Z, Nie F, Chang X, Yang Y, Zhang C, Sebe N (2018b) Dynamic affinity graph construction for spectral clustering using multiple features. IEEE Trans Neural Netw Learn Syst 29(12):6323-6332

Li Z, Yao L, Chang X, Zhan K, Sun J, Zhang H (2019) Zero-shot event detection via event-adaptive concept relevance mining. Pattern Recogn 88:595-603

Masood Z, Samar R, Raja MAZ (2019) Design of a mathematical model for the Stuxnet virus in a network of critical control infrastructure. Comput Secur 87:101565

Masood Z, Samar R, Raja MAZ (2020) Design of fractional order epidemic model for future generation tiny hardware implants. Futur Gener Comput Syst 106:43-54

Masood Z, Raja MAZ, Chaudhary NI, Cheema KM, Milyani AH (2021) Fractional dynamics of stuxnet virus propagation in industrial control systems. Mathematics 9(17):2160

Mehmood A, Zameer A, Ling SH, ur Rehman A, Raja MAZ (2020) Integrated computational intelligent paradigm for nonlinear electric circuit models using neural networks, genetic algorithms and sequential quadratic programming. Neural Comput Appl 32(14): 10337-10357

Munir A, Manzar MA, Khan NA, Raja MAZ (2019) Intelligent computing approach to analyze the dynamics of wire coating with Oldroyd 8-constant fluid. Neural Comput Appl 31(3):751-775

Muratori S, Rinaldi S (1992) Low- and high-frequency oscillations in three-dimensional food chain system. SIAM J Appl Math 52(6):1688-1706

Naz S, Zameer A, Raja MAZ, Muhammad K (2021) Weighted differential evolution heuristics for improved multilayer piezoelectric transducer design. Appl Soft Comput 113:107835

Nie F, Zhao X, Wang R, Li X, Li Z (2020) Fuzzy K-means clustering with discriminative embedding. IEEE Trans Knowl Data Eng. https://doi.org/10.1109/TKDE.2020.2995748

Nisar K, Sabir Z, Zahoor Raja MA, Ibrahim AAA, Mahmoud SR, Balubaid M, Rawat DB, Rodrigues JJ (2021) Numerical study of the environmental and economic system through the computational heuristic based on artificial neural networks. Sensors 21(19):6567

Piller O, Elhay S, Deuerlein JW, Simpson AR (2020) A content-based active-set method for pressure-dependent models of water distribution systems with flow controls. J Water Resour Plan Manag 146(4):04020009

Raja MAZ, Umar M, Sabir Z, Khan JA, Baleanu D (2018) A new stochastic computing paradigm for the dynamics of nonlinear singular heat conduction model of the human head. Eur Phys J plus 133(9):1-21

Raja MAZ, Mehmood J, Sabir Z, Nasab AK, Manzar MA (2019) Numerical solution of doubly singular nonlinear systems using neural networks-based integrated intelligent computing. Neural Comput Appl 31(3):793-812

Raja MAZ, Mehmood A, Khan AA, Zameer A (2020) Integrated intelligent computing for heat transfer and thermal radiation-based two-phase MHD nanofluid flow model. Neural Comput Appl 32(7):2845-2877

Rinaldi S, Bo SD, Nittis ED (1996) On the role of body size in a tritrophic metapopulation model. J Math Biol 35(2):158-176

Sabir Z, Manzar MA, Raja MAZ, Sheraz M, Wazwaz AM (2018) Neuro-heuristics for nonlinear singular Thomas-Fermi systems. Appl Soft Comput 65:152-169
Sabir Z, Raja MAZ, Guirao JL, Shoaib M (2020a) A neuro-swarming intelligence-based computing for second order singular periodic non-linear boundary value problems. Front Phys 8:224

Sabir Z, Raja MAZ, Shoaib M, Aguilar JG (2020b) FMNEICS: fractional Meyer neuro-evolution-based intelligent computing solver for doubly singular multi-fractional order Lane-Emden system. Comput Appl Math 39(4):1-18

Sabir Z, Sakar MG, Yeskindirova M, Saldir O (2020c) Numerical investigations to design a novel model based on the fifth order system of Emden-Fowler equations. Theor Appl Mech Lett 10:333-342

Sabir Z, Raja MAZ, Baleanu D et al (2021a) Design of Gudermannian Neuroswarming to solve the singular Emden-Fowler nonlinear model numerically. Nonlinear Dyn 106:3199-3214. https://doi. org/10.1007/s11071-021-06901-6

Sabir Z, Nisar K, Raja MAZ, Ibrahim AABA, Rodrigues JJ, Al-Basyouni K, Mahmoud S, Rawat DB (2021b) Design of Morlet wavelet neural network for solving the higher order singular nonlinear differential equations. Alex Eng J 60(6):5935-5947

Sabir Z, Raja MAZ, Arbi A, Altamirano GC, Cao J (2021c) Neuroswarms intelligent computing using Gudermannian kernel for solving a class of second order Lane-Emden singular nonlinear model. AIMS Math 6(3):2468-2485

Sabir Z, Raja MAZ, Baleanu D (2021d) Fractional Mayer Neuro-swarm heuristic solver for multi-fractional Order doubly singular model based on Lane-Emden equation. Fractals 29(5):2140017-2141219

Sabir Z, Raja MAZ, Guirao JL, Shoaib M (2021e) A novel design of fractional Meyer wavelet neural networks with application to the nonlinear singular fractional Lane-Emden systems. Alex Eng J 60(2):2641-2659

Sayed S, Nassef M, Badr A, Farag I (2019) A nested genetic algorithm for feature selection in high-dimensional cancer microarray datasets. Expert Syst Appl 121:233-243

Shen C, Wang Y, Xue W, Zhang L-H (2021) An accelerated activeset algorithm for a quadratic semidefinite program with general constraints. Comput Optim Appl 78(1):1-42

Simi V, Edla DR, Joseph J, Kuppili V (2020) Parameter-free fuzzy histogram equalisation with illumination preserving characteristics dedicated for contrast enhancement of magnetic resonance images. Appl Soft Comput 93:106364

Tao Z, Huiling L, Wenwen W, Xia Y (2019) GA-SVM based feature selection and parameter optimization in hospitalization expense modeling. Appl Soft Comput 75:323-332

Uddin I, Ullah I, Raja MAZ, Shoaib M, Islam S, Zobaer M, Nisar K, Saleel CA, Alshahrani S (2021) The intelligent networks for double-diffusion and MHD analysis of thin film flow over a stretched surface. Sci Rep 11(1):1-20

Umar M, Sabir Z, Raja MAZ (2019) Intelligent computing for numerical treatment of nonlinear prey-predator models. Appl Soft Comput 80:506-524

Umar M, Raja MAZ, Sabir Z, Alwabli AS, Shoaib M (2020a) A stochastic computational intelligent solver for numerical treatment of mosquito dispersal model in a heterogeneous environment. Eur Phys J plus 135(7):1-23

Umar M, Sabir Z, Amin F, Guirao JL, Raja MAZ (2020b) Stochastic numerical technique for solving HIV infection model of CD4+ T cells. Eur Phys J plus 135(5):403

Umar M, Sabir Z, Imran A, Wahab AH, Shoaib M, Raja MAZ (2020c) The 3-D flow of Casson nanofluid over a stretched sheet with chemical reactions, velocity slip, thermal radiation and Brownian motion. Therm Sci 24(5 Part A):2929-2939

Umar M, Sabir Z, Raja MAZ, Sánchez YG (2020d) A stochastic numerical computing heuristic of SIR nonlinear model based on dengue fever. Results Phys 19:103585

Umar M, Sabir Z, Raja MAZ, Shoaib M, Gupta M, Sánchez YG (2020e) A stochastic intelligent computing with neuro-evolution 
heuristics for nonlinear SITR system of novel COVID-19 dynamics. Symmetry 12(10): 1628

Umar M, Sabir Z, Raja MAZ, Amin F, Saeed T, Guerrero-Sanchez Y (2021a) Integrated neuro-swarm heuristic with interior-point for nonlinear SITR model for dynamics of novel COVID-19. Alex Eng J 60(3):2811-2824

Umar M, Sabir Z, Zahoor Raja MA, Gupta M, Le D-N, Aly AA, Guerrero-Sánchez Y (2021b) Computational intelligent paradigms to solve the nonlinear SIR system for spreading infection and treatment using Levenberg-Marquardt backpropagation. Symmetry 13(4):618

Upadhyay RK, Iyengar S, Rai V (1998) Chaos: an ecological reality? Int J Bifurc Chaos 8(06):1325-1333
Wilson M (2019) Optimization of the radiation shielding capabilities of bismuth-borate glasses using the genetic algorithm. Mater Chem Phys 224:238-245

Yan C, Chang X, Luo M, Zheng Q, Zhang X, Li Z, Nie F (2020) Self-weighted robust LDA for multiclass classification with edge classes. ACM Trans Intell Syst Technol TIST 12(1):1-19

Yang Y, Yang B, Wang S, Liu F, Wang Y, Shu X (2019) A dynamic ant-colony genetic algorithm for cloud service composition optimization. Int J Adv Manuf Technol 102(1):355-368

Publisher's Note Springer Nature remains neutral with regard to jurisdictional claims in published maps and institutional affiliations. 